Honam Mathematical J. 33 (2011), No. 3, pp. 355-366

http://dx.doi.org/10.5831/HMJ.2011.33.3.355

\title{
IRREDUCIBLE POLYNOMIALS WITH REDUCIBLE COMPOSITIONS
}

\author{
Eunmi ChOI
}

\begin{abstract}
In this paper we investigate criteria that for an irreducible monic quadratic polynomial $f(x) \in \mathbb{Q}[x], f \circ g$ is reducible over $\mathbb{Q}$ for an irreducible polynomial $g(x) \in \mathbb{Q}[x]$. Odoni intrigued the discussion about an explicit form of irreducible polynomials $f(x)$ such that $f \circ f$ is reducible. We construct a system of infinitely many such polynomials.
\end{abstract}

\section{Introduction}

For a field $K$ with char.0, let $f(x)$ and $g(x)$ be in $K[x]$. Then $f \circ g$ is irreducible in $K[x]$ if and only if $f(x)$ is irreducible in $K[x]$ and $g(x)-\alpha$ is irreducible in $K(\alpha)[x]$ for every root $\alpha$ of $f(x)$. This is a well known property for the irreducibility of $f \circ g$ due to Capelli (refer to [4], [2]). In particular if $f(x)=x^{2}-b x+c$ is a monic quadratic polynomial then the next lemma follows immediately.

Lemma 1. If $f(x)=x^{2}-b x+c$ is irreducible in $K[x]$ with discriminant $\Delta$, then for any polynomial $g(x) \in K[x], f \circ g$ is reducible over $K$ if and only if $g(x)-\alpha=g(x)-\frac{b}{2}-\sqrt{\frac{\Delta}{4}}$ is reducible over $K(\alpha)=K\left(\sqrt{\frac{\Delta}{4}}\right)$.

The purpose of this paper is to construct irreducible quadratic polynomials $f(x)=x^{2}-b x+c \in \mathbb{Q}[x]$ explicitly such that the composition of itself is reducible. We also investigate irreducible polynomials $f$ and $g$ while $f \circ g$ is reducible.

Throughout the paper, without mentioned otherwise, we keep notations that $f(x)=x^{2}-b x+c, \alpha$ is a root of $f(x)$ in some splitting field,

Received June 28, 2011. Accepted July 16, 2011.

2000 Mathematics Subject Classification. 11D09, 12F10, $20 B 05$.

Key words and phrases. Resultant, Discriminant, Iterated polynomial.

This paper was supported by Hannam University Research Fund 2011. 
$\Delta$ is the discriminant of $f$ and $\Delta_{0}=\frac{\Delta}{4}$. Then $\alpha=\frac{1}{2}\left(b+\sqrt{b^{2}-4 c}\right)=$ $\frac{b}{2}+\frac{\sqrt{\Delta}}{2}=\frac{b}{2}+\sqrt{\Delta_{0}}$.

\section{Irreducible polynomial with reducible iterate}

In [3], it is given as an explicit example that $f(x)=x^{2}+10 x+17$ is irreducible over $\mathbb{Q}$ but $f \circ f$ is reducible over $\mathbb{Q}$. In this section we shall develop certain criterion in order to construct an infinite series of such polynomials.

Theorem 2. Let $f(x)=x^{2}-b x+c \in \mathbb{Q}[x](b \neq 0)$ be an irreducible monic polynomial. Let $U=\sqrt{(\Delta+2 b)^{2}-4 \Delta}$.

If $c=\frac{1}{4}\left(b^{2}+2 b-2 \pm \sqrt{-8 b+4+U^{2}}\right)$ and $\frac{1}{2}(\Delta+2 b \pm U) \in \mathbb{Q}^{2}$ then $f \circ f$ is reducible.

Proof. Due to Lemma $1, f \circ f$ is reducible if and only if $f(x)-\alpha$ is reducible over $\mathbb{Q}(\alpha)$, i.e., the discriminant $\Delta(f(x)-\alpha)$ belongs to $\mathbb{Q}(\alpha)^{2}=\mathbb{Q}(\sqrt{\Delta})^{2}$. Moreover since $f(x)-\alpha=x^{2}-b x+c-\frac{b}{2}-\frac{\sqrt{\Delta}}{2}$,

$$
\Delta(f(x)-\alpha)=b^{2}-4 c+2 b+2 \sqrt{\Delta}=\Delta+2 b+2 \sqrt{\Delta} \in \mathbb{Q}(\sqrt{\Delta})^{2}
$$

so we may write $\Delta+2 b+2 \sqrt{\Delta}=(s+t \sqrt{\Delta})^{2}$ for some $s, t \in \mathbb{Q}$. Then

$$
\Delta+2 b=s^{2}+t^{2} \Delta \text { and } s t=1 .
$$

Hence $t=\frac{1}{s}$ and $\Delta+2 b=s^{2}+\frac{\Delta}{s^{2}}$. Thus we have a quartic polynomial on $s$ that $s^{4}-(\Delta+2 b) s^{2}+\Delta=0$ so

$$
s^{2}=\frac{1}{2}\left(\Delta+2 b \pm \sqrt{(\Delta+2 b)^{2}-4 \Delta}\right) .
$$

If $(\Delta+2 b)^{2}-4 \Delta=0$ then $\Delta=\left(\frac{\Delta+2 b}{2}\right)^{2}$. If $\Delta+2 b=0$ then $0=\Delta=-2 b$, it contradicts to $b \neq 0$. Hence $\Delta+2 b \neq 0$ so $\Delta=\left(\frac{\Delta+2 b}{2}\right)^{2} \neq 0$ yields $f$ is reducible, contradict. Thus we may have

$$
(\Delta+2 b)^{2}-4 \Delta=U^{2} \text { and } s^{2}=\frac{1}{2}(\Delta+2 b \pm U)
$$

for some $0 \neq U \in \mathbb{Q}$. Moreover since

$$
U^{2}=(\Delta+2 b)^{2}-4 \Delta=16 c^{2}-8\left(b^{2}+2 b-2\right) c+\left(b^{4}+4 b^{3}\right),
$$

we have $c^{2}-\frac{1}{2}\left(b^{2}+2 b-2\right) c+\frac{1}{16}\left(b^{4}+4 b^{3}-U^{2}\right)=0$, so

$$
c=\frac{1}{2}\left(\frac{1}{2}\left(b^{2}+2 b-2\right) \pm \sqrt{\frac{1}{4}\left(b^{2}+2 b-2\right)^{2}-\frac{1}{4}\left(b^{4}+4 b^{3}-U^{2}\right)}\right)
$$




$$
=\frac{1}{4}\left(b^{2}+2 b-2 \pm \sqrt{-8 b+4+U^{2}}\right)
$$

where $-8 b+4+U^{2} \in \mathbb{Q}^{2}$. Thus by taking $c$ as above we can construct $f(x)$ such that $f \circ f$ is reducible.

The polynomial $f(x)=x^{2}+10 x+17$ in [3] meets the necessary conditions in Theorem 2. In fact since $\Delta(f)=2^{5}$,

$$
U^{2}=(\Delta+2 b)^{2}-4 \Delta=2^{4} \text { and } s^{2}=\frac{1}{2}(\Delta+2 b-U)=2^{2}
$$

are squares of integers. We note that here we take $s^{2}=\frac{1}{2}(\Delta+2 b-U)$ instead of $s^{2}=\frac{1}{2}(\Delta+2 b+U)$. Hence $f \circ f$ is should be reducible. Indeed $f \circ f(x)=x^{4}+20 x^{3}+144 x^{2}+440 x+476=\left(x^{2}+12 x+34\right)\left(x^{2}+8 x+14\right)$.

Due to Theorem 2, we are able to construct infinitely many irreducible polynomials $f(x)$ that its iterate $f \circ f$ is reducible. We will find a system of such polynomials precisely in two cases that $-8 b+4+U^{2}=0$ or $-8 b+4+U^{2} \in \mathbb{Q}^{2}$.

Example 1. Assume $-8 b+4+U^{2}=0$. Then $c=\frac{1}{4}\left(b^{2}+2 b-2\right)$. Since $U^{2}=8 b-4>0$, it should be $b>2$. Thus for the $b, c, U, s \in \mathbb{Q}$ with

$$
b>2, U^{2}=8 b-4, s^{2}=\frac{1}{2}(\Delta+2 b \pm U),
$$

we can construct a family of polynomials $f(x)=x^{2}-b x+c$ which

\begin{tabular}{|c|c|c|c|c|}
\hline$b>2$ & $8 b-4=U^{2}$ & $c=\frac{b^{2}+2 b-2}{4}$ & $\Delta=-2 b+2$ & $\frac{\Delta+2 b \pm U}{2}=s^{2}$ \\
\hline (1) 5 & $6^{2}$ & $33 / 4$ & -8 & $2^{2}$ \\
\hline (2) 113 & $30^{2}$ & $12993 / 4$ & -224 & $4^{2}$ \\
\hline (3) 613 & $70^{2}$ & $376993 / 4$ & -1224 & $6^{2}$ \\
\hline (4) 1985 & $126^{2}$ & $3944193 / 4$ & -3968 & $8^{2}$ \\
\hline
\end{tabular}
are irreducible but the 2 nd iterate are reducible. Indeed we have some examples as follows:

In the case (1), let $b=5$. Then $c=33 / 4$ so $f(x)=x^{2}-5 x+\frac{33}{4}$ is irreducible since $\Delta=-8<0$, but the composition $f \circ f$ is reducible that

$f \circ f(x)=x^{4}-10 x^{3}+\frac{73}{2} x^{2}-\frac{115}{2} x+\frac{561}{16}=\frac{1}{16}\left(4 x^{2}-12 x+11\right)\left(4 x^{2}-\right.$ $28 x+51)$.

Similarly in (2) by taking $b=113$, we have $f(x)=x^{2}-113 x+\frac{12993}{4}$ which is irreducible but reducible composition $f \circ f(x)=x^{4}-226 x^{3}+\frac{38305}{2} x^{2}-\frac{1442671}{2} x+\frac{162997185}{16}$ 


$$
=\frac{1}{16}\left(4 x^{2}-463 x+11895\right)\left(4 x^{2}-468 x+13703\right) .
$$

(3) shows that $f(x)=x^{2}-613 x+\frac{376993}{4}$ is irreducible and $f \circ f(x)=x^{4}-1226 x^{3}+\frac{1127305}{2} x^{2}-\frac{230345171}{2} x+\frac{141200843185}{16}$

$$
=\frac{1}{16}\left(4 x^{2}-2428 x+368483\right)\left(4 x^{2}-2476 x+383195\right) .
$$

And in (4), $f(x)=x^{2}-1985 x+\frac{3944193}{4}$ is irreducible and $f \circ f(x)=\frac{1}{16}\left(4 x^{2}-7908 x+3908591\right)\left(4 x^{2}-7972 x+3972111\right)$.

Besides these examples we can construct infinitely many polynomials satisfying the conditions on $c$ and $U$, which are irreducible with reducible 2nd iterate.

Example 2. Assume $-8 b+4+U^{2}=V^{2}$ for some $V \in \mathbb{Q}$. Then $8 b-4=(U+V)(U-V)>0$ and $c=\frac{1}{4}\left(b^{2}+2 b-2+V\right)$. Thus

$$
s^{2}=\frac{1}{2}(\Delta+2 b+U)=\frac{1}{2}\left(b^{2}-4 c+2 b+U\right)=\frac{1}{2}(2+U-V),
$$

so $U-V=2\left(s^{2}-1\right)$. Now for $c, U, V$ satisfying

$$
\begin{gathered}
8 b-4=(U+V)(U-V), U-V=2\left(s^{2}-1\right), \\
c=\frac{1}{4}\left(b^{2}+2 b-2+V\right), s^{2}=\frac{1}{2}(\Delta+2 b+U) \text { and }-2 b+2<V,
\end{gathered}
$$

we can construct series of $f(x)=x^{2}-b x+c$ which are irreducible but the 2 nd iterate are reducible.

\begin{tabular}{cc|cccccc}
\multicolumn{1}{c}{$b>2$} & $8 b-4=(U-V)(U+V)$ & $U-V=2\left(s^{2}-1\right)$ & $s^{2}$ & $U+V$ & $U$ & $V$ \\
\hline & & & & & & \\
$(1)$ & 8 & $\left(2^{2}\right)(3)(5)$ & $(2)(3)=2\left(2^{2}-1\right)$ & $2^{2}$ & 10 & 8 & 2 \\
$(2)$ & 11 & $\left(2^{2}\right)(3)(7)$ & $(2)(3)=2\left(2^{2}-1\right)$ & $2^{2}$ & 14 & 10 & 4 \\
$(3)$ & 14 & $\left(2^{2}\right)\left(3^{3}\right)$ & $(2)(3)=2\left(2^{2}-1\right)$ & $2^{2}$ & 18 & 12 & 6 \\
$(4)$ & 17 & $\left(2^{2}\right)(3)(11)$ & $(2)(3)=2\left(2^{2}-1\right)$ & $2^{2}$ & 22 & 14 & 8 \\
$(5)$ & 20 & $\left(2^{2}\right)(3)(13)$ & $(2)(3)=2\left(2^{2}-1\right)$ & $2^{2}$ & 26 & 16 & 10 \\
& $\cdots$ & & & & & &
\end{tabular}

In case (1), let $b=8$. Then $c=\left(b^{2}+2 b-2+V\right) / 4=20$ and $\Delta=-16<0$, so

$$
s^{2}-\frac{1}{2}(\Delta+2 b+U)=2^{2}-\frac{1}{2}(-16+16+8)=0
$$

as is expected. Thus $f(x)=x^{2}-8 x+20$ is irreducible but $f \circ f(x)=x^{4}-16 x^{3}+96 x^{2}-256 x+260=\left(x^{2}-10 x+26\right)\left(x^{2}-6 x+10\right)$ is reducible. In (2), let $b=11$. Then $c=145 / 4$ and $\Delta=-24<0$, so

$$
s^{2}-\frac{1}{2}(\Delta+2 b \pm U)=2^{2}-\frac{1}{2}(-24+22+10)=0
$$

as is expected. Hence we have an irreducible $f(x)=x^{2}-11 x+145 / 4$ with 
$f \circ f(x)=x^{4}-22 x^{3}+\frac{365}{2} x^{2}-13=\frac{1}{16}\left(4 x^{2}-52 x+175\right)\left(4 x^{2}-36 x+87\right)$. Similarly in (3), $b=14, c=57$ and $\Delta=-32<0$, so

$$
s^{2}-\frac{1}{2}(\Delta+2 b \pm U)=2^{2}-\frac{1}{2}(-32+28+12)=0 .
$$

So we have an irreducible polynomial $f(x)=x^{2}-14 x+57$ such that $f \circ f(x)=x^{4}-28 x^{3}+296 x^{2}-1400 x+2508=\left(x^{2}-12 x+38\right)\left(x^{2}-16 x+66\right)$. And in case (4), $b=17, c=329 / 4$ and $\Delta=-40<0$, so

$$
s^{2}-\frac{1}{2}(\Delta+2 b \pm U)=2^{2}-\frac{1}{2}(-40+34+14)=0,
$$

thus $f(x)=x^{2}-17 x+329 / 4$ is an irreducible polynomial such that

$$
f \circ f(x)=\frac{1}{16}\left(4 x^{2}-60 x+235\right)\left(4 x^{2}-76 x+371\right) .
$$

For $(5), b=20, c=112$ and $\Delta=-48<0$, so

$$
s^{2}-\frac{1}{2}(\Delta+2 b \pm U)=2^{2}-\frac{1}{2}(-48+40+16)=0,
$$

and $f(x)=x^{2}-20 x+112$ is an irreducible polynomial such that

$$
f \circ f(x)=\left(x^{2}-22 x+124\right)\left(x^{2}-18 x+84\right) .
$$

Therefore besides the polynomial $x^{2}+10 x+17$ in [3], we can construct infinitely many quadratic irreducible polynomials whose 2 nd iterate are reducible.

\section{Irreducible polynomial with reducible composition}

This section is devoted to investigate the nature of irreducible quadratic polynomial $f$ such that the composition $f \circ g$ is reducible while $g(x)$ is irreducible of any degree. Let $f(x)=a x^{2}-b x+c$ with a root $\alpha=\frac{b}{2 a}+\sqrt{\Delta_{0}}$ for $\Delta_{0}=\frac{\Delta}{4 a^{2}}$.

Lemma 3. Let $f(x)=a x^{2}-b x+c$ be irreducible. Then for any $g(x) \in K[x], f \circ g$ is reducible over $K$ if and only if

$$
\left\{\begin{array}{cl}
g(x)-\frac{b}{2 a} & =A(x) U(x)+\Delta_{0} B(x) V(x) \\
1 & =A(x) V(x)+B(x) U(x)
\end{array}\right.
$$

for some polynomials $A(x), B(x), U(x), V(x)$ in $K[x]$. 
Proof. Due to Lemma $1, f \circ g$ is reducible over $K$ if and only if $g(x)-\alpha=g(x)-\frac{b}{2 a}-\sqrt{\Delta_{0}}$ is reducible over $K\left(\sqrt{\Delta_{0}}\right)$. That is, we may write

$$
g(x)-\frac{b}{2 a}-\sqrt{\Delta_{0}}=\left(A(x)-\sqrt{\Delta_{0}} B(x)\right)\left(U(x)-\sqrt{\Delta_{0}} V(x)\right)
$$

for some polynomials $A(x), B(x), U(x), V(x)$ in $K[x]$. This is equivalent to

$g(x)-\frac{b}{2 a}=A(x) U(x)+\Delta_{0} B(x) V(x)$ and $1=A(x) V(x)+B(x) U(x)$.

Theorem 4. Let $f(x)=a x^{2}-b x+c \in K[x]$ be an irreducible polynomial. Then there are infinitely many $g(x) \in K[x]$ such that $f \circ g$ is reducible over $K[x]$.

Proof. Let $A(x)$ and $B(x)$ in $K[x]$ be any two relatively prime polynomials. Then $A(x) V(x)+B(x) U(x)=1$ for $U(x), V(x) \in K[x]$.

For $\Delta=b^{2}-4 a c$ and $\Delta_{0}=\frac{\Delta}{4 a^{2}}$, define a polynomial $g_{0}(x) \in K[x]$ by

$$
g_{0}(x)=A(x) U(x)+\Delta_{0} B(x) V(x)+\frac{b}{2 a},
$$

and for any $\lambda(x) \in K[x]$ we let

$$
g(x)=g_{0}(x)+\lambda(x)\left(A(x)^{2}-\Delta_{0} B(x)^{2}\right) .
$$

Then with a root $\alpha=\frac{b}{2 a}+\sqrt{\Delta_{0}}$ of $f(x)$, we have

$$
\begin{aligned}
& g_{0}(x)-\alpha=g_{0}(x)-\frac{b}{2 a}-\sqrt{\Delta_{0}} \\
= & A(x) U(x)+\Delta_{0} B(x) V(x)-\sqrt{\Delta_{0}}(A(x) V(x)+B(x) U(x)) \\
= & \left(A(x)-\sqrt{\Delta_{0}} B(x)\right)\left(U(x)-\sqrt{\Delta_{0}} V(x)\right)
\end{aligned}
$$

because $A(x) V(x)+B(x) U(x)=1$. And we also have

$$
\begin{aligned}
& g(x)-\alpha=g_{0}(x)+\lambda(x)\left(A(x)^{2}-\Delta_{0} B(x)^{2}\right)-\alpha \\
= & g_{0}(x)-\alpha+\lambda(x)\left(A(x)+\sqrt{\Delta_{0}} B(x)\right)\left(A(x)-\sqrt{\Delta_{0}} B(x)\right) \\
= & \left(A(x)-\sqrt{\Delta_{0}} B(x)\right)\left(U(x)-\sqrt{\Delta_{0}} V(x)\right) \\
& \quad+\lambda(x)\left(A(x)+\sqrt{\Delta_{0}} B(x)\right)\left(A(x)-\sqrt{\Delta_{0}} B(x)\right) \\
= & \left(A(x)-\sqrt{\Delta_{0}} B(x)\right)\left[(U(x)+\lambda(x) A(x))-\sqrt{\Delta_{0}}(V(x)-\lambda(x) B(x))\right]
\end{aligned}
$$

This shows that $g_{0}(x)-\alpha$ and $g(x)-\alpha$ are reducible over $K(\alpha)$, thus due to Capelli, both $f \circ g_{0}$ and $f \circ g$ are reducible in $K$ with infinitely many polynomials $\lambda(x)$.

Refer to [1] for Theorem 4. We shall construct examples of irreducible polynomials $f$ and $g$ such that their composition $f \circ g$ is reducible. 
Example 3. Let $f(x)=x^{2}-x+1$. Then $\Delta_{0}=-\frac{3}{4}, \alpha=\frac{1}{2}(1+i \sqrt{3})$, so $f(x)$ is irreducible over $\mathbb{Q}$. Let $A(x)=x^{2}+1$ and $B(x)=x-1$. Then it is easy to see that $\operatorname{gcd}(A(x), B(x))=1$ and

$$
A(x) V(x)+B(x) U(x)=1 \text { with } V(x)=\frac{1}{2}, U(x)=-\frac{1}{2}(x+1) .
$$

Let

$$
g_{0}(x)=A(x) U(x)+\Delta_{0} B(x) V(x)+\frac{b}{2}
$$

Then

$$
g_{0}(x)=\frac{1}{2}\left(-x^{3}-x^{2}-\frac{7}{4} x+\frac{3}{4}\right)
$$

is irreducible while

$$
\begin{aligned}
g_{0}(x)-\alpha & =-\frac{1}{8}\left(2 x^{2}+2+i \sqrt{3}(-x+1)\right)(2 x+2+i \sqrt{3}) \\
& =\left(A-\sqrt{\Delta_{0}} B\right)\left(U-\sqrt{\Delta_{0}} V\right)
\end{aligned}
$$

is reducible. Now take any polynomial $\lambda(x)$, for instance, $\lambda(x)=x+2$. Let

$$
g(x)=g_{0}(x)+\lambda(x)\left(A(x)^{2}-\Delta_{0} B(x)^{2}\right) .
$$

Then

$$
g(x)=x^{5}+2 x^{4}+\frac{9}{4} x^{3}+\frac{7}{2} x^{2}-\frac{17}{8} x+\frac{31}{8}
$$

is irreducible, while

$$
\begin{aligned}
g(x)-\alpha & =\frac{1}{8}\left(2 x^{2}+2+i \sqrt{3}(-x+1)\right)\left(4 x^{3}+8 x^{2}+2 x+6+i \sqrt{3}\left(2 x^{2}+2 x-5\right)\right) \\
& =\left(A-\sqrt{\Delta_{0}} B\right)\left[\left(U-\sqrt{\Delta_{0}} V\right)+\lambda\left(A+\sqrt{\Delta_{0}} B\right)\right]
\end{aligned}
$$

is reducible. Hence, the reducibility of $g-\alpha$ implies $f \circ g$ is reducible.

Example 4. With the same irreducible polynomial $f(x)=x^{2}-x+1$ as in Example 3, we choose a different set of relatively prime polynomials $A(x)=x^{2}+x+1$ and $B(x)=x-2$. Clearly $\operatorname{gcd}(A(x), B(x))=1$ and

$$
A(x) V(x)+B(x) U(x)=1 \text { with } V(x)=\frac{1}{7}, U(x)=-\frac{1}{7}(x+3) .
$$

Since $\Delta_{0}=-\frac{3}{4}$ and $\alpha=\frac{1}{2}(1+i \sqrt{3})$,

$$
g_{0}(x)=A(x) U(x)+\Delta_{0} B(x) V(x)+\frac{b}{2}=\frac{1}{2}\left(-x^{3}-4 x^{2}-\frac{19}{4} x+2\right)
$$

is irreducible while

$$
g_{0}(x)-\alpha=-\frac{1}{28}\left(2 x^{2}+2 x+2+i \sqrt{3}(-x+2)\right)(2 x+6+i \sqrt{3})
$$


is reducible. Take any polynomial $\lambda(x)$, for instance, $\lambda(x)=x^{2}+2$. Then

$$
g(x)=x^{6}+2 x^{5}+\frac{23}{4} x^{4}+\frac{20}{7} x^{3}+\frac{153}{14} x^{2}-\frac{75}{28} x+\frac{58}{7}
$$

while

$$
g(x)-\alpha=\frac{1}{28}\left(2 x^{2}+2 x+i \sqrt{3}(-x+2)\right)
$$

$\cdot\left(14 x^{4}+14 x^{3}+42 x^{2}+26 x+22+i \sqrt{3}\left(7 x^{3}-14 x^{2}+14 x-29\right)\right)$ is reducible. Hence $f \circ g$ is reducible.

We consider another example that involves different polynomials for $h(x)$.

Example 5. Let $f(x)=x^{2}-2 x+3$. Then $f(x)$ is irreducible over $\mathbb{Q}$ with $\Delta_{0}=-2$ and root $\alpha=1+i \sqrt{2}$. Let $A(x)=x^{2}+x+1$ and $B(x)=x^{2}+1$. Then $\operatorname{gcd}(A(x), B(x))=1$ and

$$
A(x) V(x)+B(x) U(x)=1 \text { with } V(x)=-x, U(x)=1+x .
$$

Thus

$$
g_{0}(x)=A(x) U(x)+\Delta_{0} B(x) V(x)+\frac{b}{2}=3 x^{3}+2 x^{2}+4 x+2
$$

while

$$
g_{0}(x)-\alpha=\frac{1}{3}\left(3 x^{2}+x+3+i \sqrt{2} x\right)(3 x+1-i \sqrt{2})
$$

reducible. Now take any $\lambda(x)$, for example a cubic $\lambda(x)=x^{3}+1$. Then

$$
g(x)=3 x^{7}+2 x^{6}+7 x^{5}+5 x^{4}+8 x^{3}+9 x^{2}+6 x+5
$$

while

$$
\begin{aligned}
g(x)-\alpha= & \frac{1}{3}\left(3 x^{2}+x+3+i \sqrt{2} x\right) \\
\cdot & \left(3 x^{5}+x^{4}+3 x^{3}+3 x^{2}+4 x+4+i \sqrt{2}\left(-x^{4}-x-1\right)\right)
\end{aligned}
$$

reducible. Hence $f \circ g$ is reducible.

This shows that, for a given irreducible quadratic polynomial $f(x)$, it can be constructed infinitely many polynomials $g(x)$ that makes $f \circ g$ is reducible, by taking any two relatively prime polynomials. The next theorem provides more explicit criteria for the irreducibility of composition polynomials when $g(x)$ is quadratic.

Theorem 5. Let $f(x)=x^{2}-b x+c$ irreducible and $g(x)=x^{2}+A x+B$ be

$$
A=t_{0} \sqrt{-\frac{t}{s_{0}}}+s \sqrt{-\frac{s_{0}}{t}}, \quad B=s t_{0}+t s_{0} \Delta_{0}+\frac{b}{2} \quad\left(s, t, s_{0}, t_{0} \in \mathbb{Z}\right)
$$

with $\operatorname{gcd}(s, t)=1$ and $s s_{0}+t t_{0}=1$. If $-\frac{t}{s_{0}} \in \mathbb{Q}^{2}$ then $f \circ g$ is reducible over $\mathbb{Q}$. 
Proof. Let $\alpha=\frac{b}{2}+\sqrt{\Delta_{0}}$ with $\Delta_{0}=\frac{b^{2}-4 c}{4}$. Since $f(x)$ is irreducible, $f \circ g$ is reducible if and only if $g(x)-\alpha=g(x)-\frac{b}{2}-\sqrt{\Delta_{0}}$ is reducible over $\mathbb{Q}(\alpha)=\mathbb{Q}\left(\sqrt{\Delta_{0}}\right)$ by Lemma 1 . Since $g(x)$ is of degree 2 , we may factorize $g(x)-\alpha$ as

$$
\begin{aligned}
& g(x)-\frac{b}{2}-\sqrt{\Delta_{0}}=\left((r x+s)-t \sqrt{\Delta_{0}}\right)\left((u x+v)-w \sqrt{\Delta_{0}}\right) \\
= & r u x^{2}+(v r+s u) x+\left(s v+t w \Delta_{0}\right)-\sqrt{\Delta_{0}}((r w+t u) x+(s w+t v))
\end{aligned}
$$

for some $r, s, t, u, v, w \in \mathbb{Q},(r, u \neq 0)$. Thus we have

$$
g(x)-\frac{b}{2}=r u x^{2}+(v r+s u) x+\left(s v+t w \Delta_{0}\right)
$$

and $r w+t u=0$ and $s w+t v=1$. Without loss of generality we assume $g(x)$ is monic, so $u=\frac{1}{r}$ with $r \neq 0$. Hence from $r w+t u=0$, we have $r^{2} w+t=0$, i.e., $r=\sqrt{-\frac{t}{w}}$ with $-\frac{t}{w} \in \mathbb{Q}^{2}$. Moreover since $s w+t v=1$ we may consider $\operatorname{gcd}(s, t)=1$ for $s, t, v, w \in \mathbb{Z}$.

Now let $g(x)=x^{2}+A x+B$ such that

$$
A=t_{0} \sqrt{-\frac{t}{s_{0}}}+s \sqrt{-\frac{s_{0}}{t}}, \quad B=s t_{0}+t s_{0} \Delta_{0}+\frac{b}{2}
$$

such that $\operatorname{gcd}(s, t)=1$ with $s s_{0}+t t_{0}=1$ for $s_{0}, t_{0} \in \mathbb{Z}$. Then $g(x) \in$ $\mathbb{Q}[x]$. If we set $r=\sqrt{-\frac{t}{s_{0}}} \in \mathbb{Q}$ then $0=s_{0} r^{2}+t$ so

$$
0=s_{0} r+\frac{t}{r}=s_{0} \sqrt{-\frac{t}{s_{0}}}+t \sqrt{-\frac{s_{0}}{t}} .
$$

Hence with $s s_{0}+t t_{0}=1$ we have

$$
\begin{aligned}
& \left(\sqrt{-\frac{t}{s_{0}}} x+s-t \sqrt{\Delta_{0}}\right)\left(\sqrt{-\frac{s_{0}}{t}} x+t_{0}-s_{0} \sqrt{\Delta_{0}}\right) \\
= & x^{2}+\left(s \sqrt{-\frac{s_{0}}{t}}+t t_{0} \sqrt{-\frac{t}{s_{0}}}\right) x+s t_{0}+s_{0} t \Delta_{0} \\
& \quad-\sqrt{\Delta_{0}}\left(-\left(s_{0} \sqrt{-\frac{t}{s_{0}}}+t \sqrt{-\frac{s_{0}}{t}}\right) x+s s_{0}+t t_{0}\right) \\
= & x^{2}+\left(t_{0} \sqrt{-\frac{t}{s_{0}}}+s \sqrt{-\frac{s_{0}}{t}}\right) x+\left(s t_{0}+t s_{0} \Delta_{0}\right)+\frac{b}{2}-\frac{b}{2}-\sqrt{\Delta_{0}} \\
= & g(x)-\frac{b}{2}-\sqrt{\Delta_{0}}=g(x)-\alpha .
\end{aligned}
$$

Thus $g(x)-\alpha$ is reducible over $\mathbb{Q}(\alpha)$, so $f \circ g$ is reducible over $\mathbb{Q}$. 
Example 6. Choose any $s, t$ such that $\operatorname{gcd}(s, t)=1$ satisfying $s s_{0}+$ $t t_{0}=1$ and $-\frac{t}{s_{0}}=r^{2} \in \mathbb{Q}^{2}$.

\begin{tabular}{l|rrrrcrccc} 
& $f(x)$ & $s$ & $t$ & $s_{0}$ & $t_{0}$ & $r=\sqrt{-\frac{t}{s_{0}}}$ & $\Delta$ & $\Delta_{0}$ & $\alpha$ \\
\hline & & & & & & & & \\
$(1)$ & $x^{2}-x+1$ & 3 & 4 & -1 & 1 & 2 & -3 & $-\frac{3}{4}$ & $\frac{1}{2}+\sqrt{-\frac{3}{4}}$ \\
$(2)$ & $x^{2}-x+1$ & 15 & 16 & -1 & 1 & 4 & -3 & $-\frac{3}{4}$ & $\frac{1}{2}+\sqrt{-\frac{3}{4}}$ \\
$(3)$ & $x^{2}-2 x-1$ & 3 & 4 & -1 & 1 & 2 & 8 & 2 & $1+\sqrt{2}$ \\
$(4)$ & $x^{2}-2 x-1$ & 8 & 9 & -1 & 1 & 3 & 8 & 2 & $1+\sqrt{2}$ \\
& $\cdots$
\end{tabular}

In the first two cases we have the same polynomial $f(x)=x^{2}-x+1$ which is irreducible. In (1), let $s=3$ and $t=4$. Then

$$
g(x)=x^{2}+\left(r t_{0}+\frac{s}{r}\right) x+\left(s t_{0}+t s_{0} \Delta_{0}\right)+\frac{b}{2}=x^{2}+\frac{7}{2} x+\frac{13}{2}
$$

is irreducible. And of course

$$
g(x)-\alpha=\left((2 x+3)-4 \sqrt{-\frac{3}{4}}\right)\left(\left(\frac{1}{2} x+1\right)+\sqrt{-\frac{3}{4}}\right)
$$

is reducible. Moreover

$f \circ g(x)=x^{4}+7 x^{3}+\frac{97}{4} x^{2}+42 x+\frac{147}{4}=\frac{1}{4}\left(4 x^{2}+12 x+21\right)\left(x^{2}+4 x+7\right)$ is reducible. In (2), with the same $f(x)$ choose $s=15$ and $t=16$. Then

$$
g(x)=x^{2}+\frac{31}{4} x+\frac{55}{2}
$$

is irreducible but

$$
f \circ g(x)=\frac{1}{16}\left(16 x^{2}+120 x+417\right)\left(x^{2}+8 x+28\right)
$$

is reducible. In cases (3) and (4), we take another irreducible polynomial $f(x)=x^{2}-2 x-1$ with $\Delta_{0}=2$ and $\alpha=1+\sqrt{2}$. When $s=3$ and $t=4$, we have

$$
g(x)=x^{2}+\frac{7}{2} x-4
$$

is irreducible while $g(x)-\alpha=((2 x+3)-4 \sqrt{2})\left(\left(\frac{1}{2} x+1\right)+\sqrt{2}\right)$ and

$$
f \circ g(x)=\frac{1}{4}\left(4 x^{2}+12 x-23\right)\left(x^{2}+4 x-4\right)
$$

are reducible. On the other hand, by choosing $s=8$ and $t=9$, we have

$$
g(x)=x^{2}+\frac{17}{3} x-9
$$


is irreducible while $f \circ g(x)=\frac{1}{9}\left(x^{2}+6 x-9\right)\left(9 x^{2}+48 x-98\right)$ is reducible. It concludes that, for a given irreducible polynomial $f(x)$ we can construct infinitely many $g(x)$ explicitly such that $f \circ g$ is reducible.

\section{The $t$-th iterate of polynomials}

The irreducibility of 2nd iterate is based on the Capelli theorem.

Lemma 6. [4] $g \circ f$ is irreducible in $K[x]$ if and only if $g(x)$ is irreducible in $K[x]$ and $f(x)-\beta$ is irreducible in $K(\beta)[x]$ for every root $\beta$ of $g(x)$.

This can be extended to any $t$-th iterate so that we may be able to construct polynomials explicitly.

Theorem 7. Let $g(x) \in K[x]$ be irreducible. For any $f(x) \in K[x]$, $g \circ f_{t+1}$ is irreducible in $K[x]$ if and only if $f(x)-\beta_{t}$ and $f(x)-f_{j}\left(\beta_{t}\right)$ $(0 \leq j \leq t)$ are irreducible in $K\left(\beta_{t}\right)[x]$ for all roots $\beta_{t}$ of $g \circ f_{t}(x)$.

Proof. The case of $t=0$ is the Lemma 6 . When $t=1, g \circ f_{2}$ is irreducible over $K \Leftrightarrow g \circ f$ is irreducible over $K$ and $f(x)-\beta_{1}$ is irreducible over $K\left(\beta_{1}\right)$ for every root $\beta_{1}$ of $g \circ f(x) \Leftrightarrow f(x)-\beta$ is irreducible over $K(\beta)$ and $f(x)-\beta_{1}$ is irreducible over $K\left(\beta_{1}\right)$ for every roots $\beta$ of $g(x)$ and $\beta_{1}$ of $g \circ f(x)$.

For every root $\beta_{1}$ of $g \circ f, f\left(\beta_{1}\right)$ is a zero of $g(x)$. Hence if we let $\beta=f\left(\beta_{1}\right)$, then $g \circ f_{2}$ is irreducible if and only if $f(x)-f\left(\beta_{1}\right)$ is irreducible over $K\left(f\left(\beta_{1}\right)\right)$ and $f(x)-\beta_{1}$ is irreducible over $K\left(\beta_{1}\right)$ for every root $\beta_{1}$ of $g \circ f(x)$. But since $K\left(f\left(\beta_{1}\right)\right)=f\left(K\left(\beta_{1}\right)\right)=K\left(\beta_{1}\right)$, $g \circ f_{2}$ is irreducible if and only if both $f(x)-f\left(\beta_{1}\right)$ and $f(x)-\beta_{1}$ are irreducible over $K\left(\beta_{1}\right)$ for every root $\beta_{1}$ of $g \circ f(x)$.

Similarly $g \circ f_{3}$ is irreducible over $K$ if and only if $g \circ f_{2}$ is irreducible over $K$ and $f(x)-\beta_{2}$ is irreducible over $K\left(\beta_{2}\right)$ for every root $\beta_{2}$ of $g \circ f_{2}(x)$. This is equivalent to that $f(x)-f\left(\beta_{1}\right)$ and $f(x)-\beta_{1}$ are irreducible over $K\left(\beta_{1}\right)$ and $f(x)-\beta_{2}$ is irreducible over $K\left(\beta_{2}\right)$ for every roots $\beta_{1}$ of $g \circ f(x)$ and $\beta_{2}$ of $g \circ f_{2}(x)$. But every root $\beta_{2}$ of $g \circ f_{2}$ satisfies that $f\left(\beta_{2}\right)$ is a zero of $g \circ f(x)$. Hence if we let $\beta_{1}=f\left(\beta_{2}\right)$ then $g \circ f_{3}$ if irreducible if and only if $f(x)-f_{2}\left(\beta_{2}\right)$ and $f(x)-f\left(\beta_{2}\right)$ are irreducible over $K\left(f\left(\beta_{2}\right)\right)$ and $f(x)-\beta_{2}$ is irreducible over $K\left(\beta_{2}\right)$ for every root $\beta_{2}$ of $g \circ f_{2}(x)$, hence it is so if and only if $f(x)-f_{2}\left(\beta_{2}\right), f(x)-f\left(\beta_{2}\right)$ and $f(x)-\beta_{2}$ are irreducible over $K\left(\beta_{2}\right)$ for every root $\beta_{2}$ of $g \circ f_{2}(x)$.

Therefore $g \circ f_{t+1}$ is irreducible over $K$ if and only if $f(x)-f_{j}\left(\beta_{t}\right)$ $(1 \leq j \leq t)$ and $f(x)-\beta_{t}$ are irreducible over $K\left(\beta_{t}\right)$ for every root $\beta_{t}$ of $g \circ f_{t}(x)$. 
Acknowledge. I would like to thank the referee for the valuable comments.

\section{References}

[1] N. Ali, On the irreducibility for composition of polynomials. International Mathematical Forum, 4, (40), 2009, 2001-2008

[2] L. Danielson, B. Fein. On the irreducibility of iterates of $x^{n}-b$, The Americal Math. Soc. 130 (2011), 1589-1596.

[3] R.K.W. Odoni. The Galois theory of iterates and composites of polynomials, Prod. London Math Soc. (3), 51 (3), 1985, 385-414

[4] R.K.W. Odoni. On the prime divisors of the sequence $w_{n+1}=1+w_{1} \cdots w_{n}$. J. London Math. Soc. 32 (1985), 1-11.

\section{Eunmi Choi}

Department of Mathematics, HanNam University, Daejon 306-791, Korea.

E-mail: emc@hnu.kr 